\title{
Main Manuscript for \\ The terminal heme synthetic enzyme, Coproheme Decarboxylase, coordinates heme synthesis and uptake in response to iron in Mycobacteria
}

Rebecca K. Donegan ${ }^{\mathrm{a}}$, Jacqueline Copeland ${ }^{\mathrm{h}}$, Stanzin Edgha ${ }^{\mathrm{j}}$, Gabriel Brown ${ }^{\mathrm{a}}$, Owen F. Hale $^{b}$, Avishek Mitra ${ }^{k}$, Hui Yang', Harry A. Dailey ${ }^{\mathrm{d}, \mathrm{e}, \mathrm{f}}$, Michael Niederweis ${ }^{\mathrm{g}}$, Paras Jain ${ }^{\mathrm{h}, \mathrm{j} *}$, and Amit R. Reddi ${ }^{\mathrm{a}, \mathrm{b}, \mathrm{c} *}$

\section{Author Affiliations:}

aSchool of Chemistry and Biochemistry, Georgia Institute of Technology, Atlanta, GA 30332

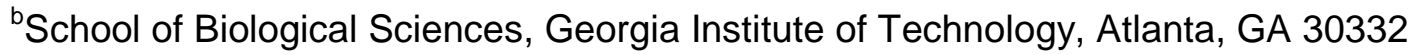

'Parker Petit Institute for Bioengineering and Biosciences, Georgia Institute of Technology, Atlanta, GA 30332

${ }^{\mathrm{d}}$ Department of Department of Microbiology, University of Georgia, Athens, Georgia, 30602

e Department of Biochemistry and Molecular Biology, University of Georgia, Athens, Georgia, 30602

fBiomedical and Health Sciences Institute, University of Georgia, Athens, Georgia, 30602

${ }^{9}$ Department of Department of Microbiology, University of Alabama at Birmingham, Birmingham, AL, 35294

${ }^{\mathrm{h}}$ Department of Microbiology and Immunology, Albert Einstein College of Medicine, Bronx, NY, 10461

'Department of Neurology, Albert Einstein College of Medicine, Bronx, NY, 10461

j Trudeau Institute, Saranac Lake, NY, 12983

k.Department of Microbiology and Molecular Genetics, Oklahoma State University, Stillwater, Oklahoma, 74078.

\section{${ }^{\star}$ Co-corresponding Authors:}

Amit R. Reddi

Georgia Institute of Technology 
School of Chemistry and Biochemistry

901 Atlantic Drive, MC0400

Atlanta, GA 30332, USA

Phone: (404) 385-1428

E-mail: amit.reddi@chemistry.gatech.edu

Paras Jain

Trudeau Institute

154 Algonquin Ave,

Saranac Lake, NY 12983

Phone: (646) 920- 2041

E-mail: Parasjain29@gmail.com

Paste ORCIDs (if any) here. Please note individual authors must also link their ORCID account to their PNAS account at www.pnascentral.org.

\section{Classification}

Biological sciences : Biochemistry

\section{Keywords}

Mycobacterium tuberculosis, Mycobacterium smegmatis, heme homeostasis, iron homeostasis, heme synthesis

\section{Author Contributions}

R.K.D., J.C., S.I, G.B., O.H., P.J., H.Y. performed research and analyzed data. H.A.D., A.M, M.N. provided key reagents and designed research. R.K.D., P.J., and A.R.R. designed research, analyzed data and wrote the paper, with input from all authors.

This PDF file includes:

Main Text

Figures 1 to 4 


\begin{abstract}
Heme is both an essential cofactor and an abundant source of nutritional iron for the human pathogen Mycobacterium tuberculosis (Mtb). While heme is required for Mtb survival and virulence, it is also potentially cytotoxic. Since Mtb has the ability to both make and uptake heme, the de novo synthesis of heme and its acquisition from the host must be balanced in order to mitigate heme toxicity. However, the mechanisms employed by Mtb to regulate heme uptake, synthesis, and bioavailability are poorly understood. By integrating ratiometric heme sensors with mycobacterial genetics, cell biology, and biochemistry, we determined that the terminal heme biosynthetic enzyme, coproheme decarboxylase $(\mathrm{ChdC})$, plays a role in regulating both heme bioavailability and uptake in Mtb. Moreover, we found that Mtb has a preference for scavenging reduced ferrous heme and exhibits a cell surface heme reductase activity that is regulated by ChdC. In Mtb, ChdC expression is down-regulated when iron is limiting, which in-turn increases both heme import and bioavailability. Such a mechanism may serve to protect cells from heme toxicity while trying to meet the nutritional demand for iron. Our results demonstrate that heme synthesis and uptake are tightly integrated in mycobacteria and represent the first example of a heme synthetic enzyme playing a role in controlling heme uptake.
\end{abstract}

\title{
Significance Statement
}

Heme is an essential but potentially cytotoxic cofactor and iron source for the pathogen, Mycobacterium tuberculosis (Mtb). To understand how Mtb coordinates heme 
uptake and synthesis to mitigate heme toxicity, we integrated heme sensors with mycobacterial genetics and biochemical approaches to probe the interplay between heme synthesis and scavenging. We discovered that the terminal heme synthetic enzyme, coproheme decarboxylase (ChdC), negatively regulates heme uptake and utilization in response to iron availability through a mechanism involving control of a ferric heme reductase. During iron limitation, ChdC is downregulated, thereby enhancing exogenous heme reduction, uptake and utilization while simultaneously suppressing heme synthesis, which allows Mtb to avoid heme toxicity. Our results highlight the close coordination between heme synthesis and uptake in mycobacteria.

\section{Main Text}

\section{Introduction}

Heme is both an essential cofactor and a primary source of iron for the human pathogen Mycobacterium tuberculosis (Mtb) $(1,2)$. As a cofactor, heme enables a number of physiological functions, including respiration, gas sensing, and protection against reactive oxygen and nitrogen species generated by the host immune system. Nutritionally, heme is the most bioavailable source of iron in the human host, with more than two-thirds of iron in circulation found bound to hemoglobin as heme-iron (3). Although heme is essential for Mtb, which can both make and scavenge heme, it is also potentially cytotoxic if present in excess or mishandled by cells $(4,5)$. As a consequence, Mtb must tightly regulate heme synthesis, import, and bioavailability in order to mitigate heme toxicity. However, the mechanisms underlying the regulation of heme homeostasis in mycobacteria are poorly understood $(1,6,7)$. 
At least two unique heme uptake pathways have been identified in Mtb; one that requires albumin for heme uptake and one that is albumin independent.(7-9) The presence of multiple pathways for heme scavenging implies that heme uptake is of great importance for Mtb virulence. Indeed, the knockout of the albumin independent pathway alone decreases survival in the macrophage.(8) While the factors that govern heme uptake in Mtb are largely unknown, heme and iron uptake in Mtb are regulated independently.(10)

In addition to uptake, Mtb encodes a complete heme biosynthetic pathway that begins with the synthesis of 5 -aminolevulinic acid $(A L A)$ from glutamate via the $C_{5}$ pathway (11). ALA is then metabolized to coproporphyrinogen III in 4 enzymatic steps which are common among eukaryotes. However, unlike eukaryotes and gram-negative bacteria, which utilize the canonical protoporphyrin dependent branch (PPD) for the three terminal heme synthesis steps, Mtb synthesizes heme through the coproporphyrin dependent branch (CPD).(11-13) In the PPD branch, coproporphyrinogen III is oxidatively decarboxylated to protoporphyrinogen IX, followed by oxidation to protoporphyrin IX and insertion of iron by ferrochelatase to create heme.(11) In the CPD branch utilized by Mtb, coproporphyrinogen III is first oxidized to coproporphyrin III, followed by ferrochelatase-catalyzed iron insertion to make coproheme III and finally decarboxylation to make heme. The coproporphyrinogen oxidizing enzyme and ferrochelatase are homologous in the two pathways, despite binding different substrates.(11) However the terminal enzyme in Mtb, coproheme decarboxylase (ChdC), is unique to the CPD branch. (11) The divergence of heme synthesis strategies in Mtb and the necessity of heme for Mtb survival has led to the consideration of targeting heme synthesis for anti-Mtb therapies. $(6,13,14)$ However, it is unclear what the relative 
contribution of exogenously scavenged and endogenously synthesized heme is towards filling the bioavailable heme pool and meeting the metabolic demands of Mtb. Moreover, given that in some organisms, such as the eukaryote Saccharomyces cerevisiae, heme import is upregulated when heme synthesis is ablated, (15) it is not known what impact perturbing heme synthesis may have on heme uptake pathways in mycobacteria.

Herein, by integrating genetically encoded ratiometric heme sensors with mycobacterial molecular genetics and biochemical assays, we probe the mechanisms underlying the coordination of heme uptake, synthesis, and bioavailability in Mtb and Mycobacterium smegmatis (Msm). Our results establish that both Mtb and Msm maintain a reservoir of exchangeable bioavailable heme and that de novo synthesized heme is more bioavailable than exogenously supplied heme. Moreover, we determine that heme uptake and utilization is negatively regulated by the terminal heme biosynthetic enzyme, ChdC. Msm and Mtb strains with a knockout of chdC significantly increase both the uptake and utilization of exogenous heme in both Msm and Mtb. Furthermore, Mtb ChdC expression is proportional to iron availability. When Mtb is iron limited, ChdC expression is downregulated, which in turn increases the uptake and utilization of exogenous heme. Our results further indicate that Mtb has a preference for taking up reduced ferrous heme and exhibits a cell surface heme reductase activity that is inhibited by ChdC. Altogether, ChdC coordinates the synthesis and uptake of heme in response to iron availability, providing a means to prevent heme toxicity while trying to meet the nutritional demand for iron using heme uptake pathways.

\section{Results}

Characterization of heme bioavailability in $M$. smegmatis and M. tuberculosis. Total cellular heme can be considered as the sum of exchange inert and labile heme 
(LH) $(1,4,5,16)$. The majority of intracellular heme is exchange inert, corresponding to heme that is tightly bound to hemoproteins. A smaller fraction of intracellular heme is maintained as a LH pool that is bioavailable for heme dependent processes. To measure LH within mycobacteria, we incorporated and validated previously described genetically encoded LH sensors (16-20) in Msm and Mtb (Supplemental Appendix and Fig. S1 and S2). Heme sensor 1 (HS1) is a tri-domain construct consisting of the heme binding protein cytochrome $b_{562}\left(C y t b_{562}\right)$, fused to enhanced green fluorescent protein (eGFP), and monomeric Katushka red fluorescent protein 2 (mKATE2). The fluorescence of eGFP is quenched upon heme binding to $C y t b_{562}$, whereas the mKATE2 fluorescence is unaffected.(16) Thus, the eGFP/mKATE2 fluorescence ratio is inversely proportional to cellular LH.(16)

In WT Msm, both the high affinity prototype sensor HS1, which exhibits dissociation constants of $3 \mathrm{nM}$ for ferric heme $\left(K_{\mathrm{D}}^{\mathrm{Fe}(\mathrm{III})}\right)$ and $\sim 1 \mathrm{pM}$ for ferrous heme $\left(K_{\mathrm{D}}^{\mathrm{Fe}(\mathrm{II})}\right)(16,17)$, and a moderate affinity sensor variant $\mathrm{HS} 1-\mathrm{M} 7 \mathrm{~A}$, which exhibits $K_{\mathrm{D}}^{\mathrm{Fe}(\mathrm{III})}$ $=2 \mu \mathrm{M}$ and $K_{\mathrm{D}}{ }^{\mathrm{Fe}(\mathrm{II})}=25 \mathrm{nM}$, (16) exhibit a dose-dependent increase in sensor eGFP/mKATE2 fluorescence ratio in response to succinylacetone (SA), an inhibitor of the heme biosynthetic enzyme porphobilinogen synthase (Fig. S1 a-b).(21) In contrast, HS1-M7A,H102A, which cannot bind heme and serves as a control for hemeindependent changes to sensor fluorescence, did not exhibit SA-dependent changes in eGFP/mKATE2 fluorescence ratios (Fig. S1c). Together, these data indicated that both HS1 and HS1-M7A were competent for sensing intracellular LH in Msm. By adapting previously established sensor calibration procedures to Msm (Fig. S1 d-f, see Supplemental Appendix) we estimated that the heme occupancies of HS1 and HS1-M7A were $>85 \%$ and $\sim 50 \%$, respectively, making HS1-M7A ideally suited for measuring LH 
in Msm. In the avirulent Mtb strain $\mathrm{mc}^{2} 6230$ (WT Mtb), the heme sensors HS1 and HS1-M7A likewise exhibited heme-dependent fluorescence responses and the control, HS1-M7A, H102A, did not (Fig. S2 a-c). The in situ calibration of the sensors in Mtb (Fig. S2 d-f) revealed that HS1 is saturated with heme, like in Msm, and HS1-M7A is < $20 \%$ bound to heme, unlike in Msm where HS1-M7A is $50 \%$ bound to heme. LH was only completely depleted from HS1 after repeated exposure to $500 \mu \mathrm{M} \mathrm{SA}$, which was applied every 72 hours for 13 days (Fig. S2 b). Both exogenous hemin chloride (referred to hereafter as heme) (Fig. S2 a,c) and hemoglobin (Hb) (Fig. S2c) significantly increased HS1-M7A detected LH, which indicated that scavenged heme contributed to the LH pool. The heme biosynthetic precursor ALA had little effect on the LH pool of Mtb (Fig. S2c), which indicated that heme synthesis and/or partitioning of synthesized heme into the LH pool was tightly regulated. The observation that Msm has a greater concentration of bioavailable heme than Mtb, as assessed by HS1-M7A heme loading, is not due to generally higher levels of heme in Msm. In fact, Mtb had > 3-fold higher heme per cell (Fig. S2g) or nearly 7 times more heme per milligram of protein than Msm (Fig. S2h). Therefore, the reduced LH levels of Mtb result from differences in heme speciation and buffering between Mtb and Msm.

Exogenous vs endogenous heme utilization in $\boldsymbol{M}$. smegmatis. Since mycobacteria can both synthesize and uptake heme, $(11,22)$ we sought to determine if there was a difference in the bioavailability and utilization of exogenously supplied versus endogenously synthesized heme. Towards this end, we generated a heme auxotrophic strain of Msm with a deletion of the first heme synthesis enzyme, glutamyl tRNA reductase (GtrR) (11). $\Delta g t r R$ Msm exhibited a heme auxotrophy (Fig. 1a, black open squares with cyan line), and supplementation with $5 \mu \mathrm{g} / \mathrm{mL}$ ALA, which restored heme 
synthesis (Fig. 1b, gray bars), rescued growth to that of WT Msm (Fig. 1a, orange triangles and black circles, respectively). However, $\Delta g t r R$ Msm supplemented with 50 $\mu \mathrm{M}$ exogenous heme exhibited a substantial growth defect (Fig. 1a, red diamonds) compared to WT Msm (Fig. 1a, black circles) and $\Delta g t r R$ Msm supplemented with ALA (Fig. 1a, orange triangles). This growth defect was not due to heme toxicity as WT Msm supplemented with $50 \mu \mathrm{M}$ heme (Fig. 1a, blue squares) had similar growth rates to untreated cells (Fig. 1a, black circles). These growth data indicated that Msm did not utilize exogenous heme as efficiently as endogenously synthesized heme.

To determine if the growth disparity between endogenous and exogenous heme utilization corresponded to differences in intracellular heme accumulation and/or bioavailability, we measured total (Fig. 1b) and labile (Fig. 1c) heme in $\Delta$ gtrR Msm cells supplemented with heme or ALA. $\Delta g t r R$ Msm cells were starved of heme and ALA for 18 hours, which was sufficient to reduce total heme (Fig. 1b) and deplete LH (Fig. 1c). $\Delta g t r R$ Msm cells were then supplied with either $5 \mu \mathrm{g} / \mathrm{mL}$ ALA for endogenous heme synthesis, or $50 \mu \mathrm{M}$ heme as a source of exogenous heme. Both ALA and heme supplementation restored total heme to a similar extent in $\Delta g t r R$ Msm cells over a broad time range (Fig. 1b). However, ALA more rapidly increased the bioavailable LH pool than exogenously supplied heme over the same time span (Fig. 1c). ALA supplementation resulted in a significant increase in LH within 2 hours, whereas it took more than 8 hours for exogenously supplied heme to have the same effect. Therefore, while both exogenous and endogenous heme contributed to intracellular heme accumulation, exogenous heme did not populate the LH pool as rapidly and was less bioavailable, thereby explaining its poor rescue of $\Delta g t r R$ cells. 
The heme dependent catalase-peroxidase KatG plays an important role in detoxifying reactive oxygen species in mycobacteria and is important for mycobacterial virulence.(23) To determine if the difference in utilization of exogenous versus biosynthesized heme affected the activity of this hemoprotein, we measured KatG activity in $\Delta g t r R$ Msm using an in-gel catalase-peroxidase activity assay.(24, 25) We found that while endogenously synthesized heme rescued KatG activity, exogenous heme did not (Fig. 1d). Upon depletion of heme, $\Delta g t r R$ Msm did not have active KatG (Fig. 1d, time 0). Supplementation of $\Delta g t r R$ Msm with ALA yielded active KatG within 4 hours(Fig. 1d) However, heme supplementation did not yield active KatG even after 24 hours.(Fig. 1d) Altogether, these results revealed that while $\Delta$ gtrR Msm utilized exogenous heme for growth and metabolism, it was less bioavailable than endogenously synthesized heme.

\section{ChdC is a negative regulator of heme uptake and utilization in $M$.} smegmatis. The terminal heme synthesis enzyme ChdC is unique to actinobacteria and firmicutes and had been put forth as a potential target for anti-Mtb therapies.(13) The poor ability of $\Delta g t r R$ Msm to utilize exogenously supplied heme led us to speculate that heme would be relatively ineffective at rescuing Msm lacking chdC. However, unlike in $\Delta g t r R$ cells, we found that $\Delta c h d C$ Msm was fully rescued by exogenous heme (Fig. 1a, green triangles). To better understand the relationship between the heme synthetic enzymes GtrR and ChdC and heme uptake, bioavailability and utilization, we measured total heme, $\mathrm{LH}$, and KatG activity in heme depleted $\Delta g t r R$ and $\Delta$ chdC Msm cells supplemented with exogenous heme. Heme supplementation resulted in a four-fold increase in total heme accumulation after 10 hours in $\Delta c h d C$ cells compared to $\Delta g t r R$ cells, which suggested that $\mathrm{ChdC}$ may play a role in regulating heme uptake (Fig. 1e). 
Strikingly, unlike in $\Delta g t r R$ cells, exogenous heme resulted in substantially elevated LH in $\Delta c h d C$ cells (Fig. 1f). At 2 and 6 hours after the addition of exogenous heme, when total heme is similar between $\Delta c h d C$ and $\Delta g t r R$ Msm, LH was significantly elevated in $\Delta c h d C$ Msm. Moreover, exogenous heme activated KatG in $\Delta c h d C \mathrm{Msm}$, but not in $\Delta g t r R \mathrm{Msm}$ (Fig. 1g). and $\triangle$ chdC cells accumulated $\sim 7$-fold more of the fluorescent heme analog zinc mesoporphyrin (ZnMP) than WT and $\Delta g t r R$ cells, which internalized similar levels of ZnMP (Fig 1h, and Fig S3a). Taken together, our results indicated that heme biosynthetic enzymes play a substantial role in regulating the uptake and utilization of exogenous heme.

However, it was unclear if heme uptake and bioavailability was positively regulated by GtrR or negatively regulated by ChdC. To differentiate these possibilities, we assayed KatG activity, as a reporter of heme uptake and availability, in a $\Delta g t r R$ $\Delta c h d C$ double knockout. The double knockout had KatG activity similar to $\triangle c h d C$ cells when supplemented with exogenous heme (Fig. S3b,c), which implied that the increased heme utilization in $\triangle c h d C \mathrm{Msm}$ was due to the loss of ChdC, not the presence of GtrR. Moreover, these results also indicated that any potential build-up of heme biosynthetic precursors in $\triangle c h d C$ cells did not contribute to the elevated heme uptake and utilization observed in $\triangle c h d C$ cells. Additionally, the expression of catalytically inactive mutants of Mtb ChdC did not inhibit heme uptake (See Supplemental Appendix and Fig. S4), suggesting a role for ChdC-mediated coproheme decarboxylation, heme binding, or heme transfer to downstream targets in regulating heme uptake and utilization. Altogether, our results indicated that $\mathrm{ChdC}$ was a negative regulator of heme uptake in Msm. 
ChdC is a negative regulator of heme uptake and utilization in M. tuberculosis. To establish the role of ChdC as a negative regulator of heme uptake and utilization in Mtb, we generated heme auxotrophic Mtb strains with deletions of $g t r R$ or $c h d C$ and tested their ability to utilize exogenous heme. Both $\Delta g t r R$ and $\Delta c h d C$ Mtb exhibited a heme auxotrophy (Fig. S5). As with Msm, ALA rescued $\Delta$ gtrR Mtb cells more efficiently than exogenous heme. However, unlike Msm, $\Delta$ gtrR Mtb + ALA (Fig. 2a, green triangles) did not grow as efficiently as WT Mtb (Fig. 2a, purple circles), which suggested that $\Delta$ gtrR Mtb cannot import ALA in a manner that fully supports growth in the indicated culture conditions. Moreover, $\triangle$ chdC Mtb supplemented with exogenous heme (Fig. 2a, red diamonds) grew at a rate similar to WT Mtb and faster than heme supplemented $\Delta g t r R$ cells (Fig. 2a, orange triangles). Additionally, $\Delta c h d C$ cells accumulated more ZnMP than WT and $\Delta$ gtrR Mtb cells (Fig. 2b,c), which internalized similar levels of ZnMP. The 48hour timepoint where we observed a $\sim 50 \%$ increase in ZnMP uptake in $\triangle$ chdC cells compared to WT, was approximately two doubling times for Mtb. This timepoint corresponded to the 10-hour timepoint for Msm (Fig. 1h), which had a doubling time of approximately 5 hours. Similar to heme uptake in $\Delta c h d C$ Msm (Fig. 1b), early time points for Mtb ZnMP uptake did not exhibit increased ZnMP uptake in $\Delta c h d C$ Mtb (Fig. S6).

Heme dependent transcriptional responses in M. tuberculosis. In order to assess the status of heme metabolism and utilization between de novo synthesized and exogenously scavenged heme, as well as ChdC-regulated heme uptake, we examined the expression of a wide-panel of heme homeostatic genes in response to heme in WT, $\Delta g t r R$, and $\triangle c h d C$ cells using RT-qPCR. Markers for heme metabolism included transcripts encoding genes for heme biosynthetic enzymes, e.g. gtrR, gsaM, cpfC, urod 
and $c h d C,(11)$ heme catabolizing enzymes e.g. mhuD and bvr,(26, 27) heme transport proteins, e.g. ppe36, ppe37, ppe62, $d p p A, d p p D$ and $m m p / 3,(7-9,28)$ additional factors previously implicated in heme uptake, e.g. fix $A$, fixB, $m m p / 11,(9,10)$ iron storage proteins, bfrA and $b f r B,(29)$ a heme-dependent peroxidase, $k a t G,(30)$ and an iron homeostatic factor, ideR.(31) For heme treatments, WT, $\Delta$ gtrR and $\Delta c h d C$ Mtb cells were cultured in $7 \mathrm{H} 9+$ albumin dextrose and salt (7H9+ADS, see methods) supplemented with $25 \mu \mathrm{M}$ heme until growth reached saturation. Cells were then either washed and diluted into 7H9+ADS without heme for 3 days ("heme depleted") or diluted into $25 \mu \mathrm{M}$ heme for 3 days. $\Delta g$ trR Mtb + ALA cells were cultured in 7H9+ADS supplemented with $5 \mu \mathrm{g} / \mathrm{mL}$ ALA. As a control, untreated WT Mtb cells were continuously cultured in $7 \mathrm{H} 9+\mathrm{ADS}$ and served as a reference to evaluate heme status in all mutants and growth conditions.

In WT Mtb, exogenous heme supplementation regulates the expression of the early and late enzymes of the heme biosynthetic pathway distinctly, with heme causing a 4-fold decrease in gtrR transcript and a 2.5-fold increase in chdC transcript (Fig. 2d and Dataset S1). The differential effects of exogenous heme on $g t r R$ and $c h d C$ expression suggests ChdC plays additional roles that extend beyond heme synthesis, including regulating heme uptake and bioavailability. Additionally, heme treatment of WT Mtb upregulated only the cell surface heme transport proteins ppe37 and ppe62, while other heme uptake proteins ( $p p e 36, d p p A$, and $d p p D$ ) had little to no change in transcript, or in the case of $\mathrm{mmp} / 3$, had decreased expression. Exogenous heme downregulated the expression of the heme degrading heme oxygenase, mhuD, and the ferritin, bfrB, possibly indicating that heme degradation to release iron may not be occurring to an appreciable extent. Of note, WT Mtb cells depleted of exogenous heme showed 
lingering effects of heme exposure in their transcriptional profiles (Fig. 2d, column 2). This may be due to the continued internalization of recalcitrant heme retained at the cell surface even after washing and removal of exogenous heme from the cells and culture media.

Heme depleted $\Delta g t r R$ cells exhibited a transcriptional profile consistent with a heme starvation state, with all transcripts encoding heme biosynthetic and uptake proteins being induced (Fig. 2d). Supplementing $\Delta g t r R$ cells with ALA, but not exogenous heme, generated a heme replete state, with expression of heme synthesis and transport genes being repressed or restored relative to WT (Fig. 2d). The heme vs. ALA dependent transcriptional responses in $\Delta g t r R$ cells are consistent with endogenous heme being more bioavailable than exogenously supplied heme (Fig. 2d and Fig. S7a). Interestingly, restoring heme synthesis in $\Delta g t r R$ cells with ALA resulted in iron limitation, as inferred from the $\sim 3$-fold decrease in ide $R$ and $b f r B$, iron homeostatic genes that positively correlate with iron levels. $(29,32)$ Consistent with the notion that heme synthesis can limit iron, heme depleted $\Delta g t r R$ cells, which cannot synthesize heme, have a 2 -fold increase in $i d e R$ and 1.5 -fold increase in $b f r B$, indicating a more iron replete state relative to WT cells. Although exogenous heme does not rescue the heme deficiency of $\Delta g t r R$ cells, it does serve to repress $b f r B$, indicating heme can alter cellular iron homeostasis (Fig. 2d and Fig. S7b), albeit through an unknown mechanism. Surprisingly, mhuD expression did not correlate with heme levels as one might expect, with heme depleted $\Delta g t r R$ cells exhibiting elevated mhuD transcript and ALA or heme supplemented $\Delta g t r R$ cells exhibiting depressed $m h u D$ levels. Altogether, transcriptomic profiling indicates that ALA, but not exogenous heme, effectively alleviates the heme 
deficiency of $\Delta g t r R$ cells, and that heme synthesis, and exogenous heme to a certain degree, can contribute to limiting cellular iron.

Surprisingly, heme depleted $\triangle c h d C$ cells do not exhibit many of the transcriptional hallmarks of heme starvation observed in heme depleted $\Delta g t r R$ cells, including the induction of heme biosynthetic and transport genes (Fig. 2d). In fact, heme depleted $\triangle$ chdC Mtb had a similar transcriptional response to heme depleted WT Mtb, with one exception being $\mathrm{mmp} / 3$, which had a $>2$-fold reduction in $\Delta c h d C$ cells compared to WT (Fig. 2d). Transcript levels in heme treated $\Delta$ chdC cells were very similar to heme treated WT cells (Fig. 2d and Fig. S7c). One exception was the regulation of the heme biosynthesis gene $u r o D$, which was upregulated in WT Mtb treated with heme but did not have altered transcription in heme treated $\Delta c h d C$ Mtb (Fig. 2d). Given that $c h d C$ and $u r o D$ are predicted to be in the same operon, it is possible the loss of $c h d C$ alters expression of uroD through an undiscovered regulatory mechanism or due to differences in intracellular heme in $\Delta c h d C$ cells compared to WT cells. Additionally, the transcript levels of the iron homeostasis genes ide $R$ and $b f r B$ were decreased $\geq 2$-fold in heme treated $\triangle$ chdC compared to heme treated WT Mtb (Fig. 2d and Fig. S7c). Since bfrB is repressed in response to exogenous heme in WT Mtb (Fig. 2d), the more severe reduction of $b f r B$ in heme treated $\triangle c h d C$ cells compared to WT is consistent with the observation that heme uptake is increased in $\Delta c h d C$ cells. Notably, none of the previously characterized heme uptake proteins were strongly induced in heme depleted $\Delta$ chdC cells relative to WT or $\Delta g t r R$ strains, indicating that $\mathrm{ChdC}$ regulation of heme uptake and bioavailability does not occur through the transcriptional regulation of known heme transport proteins. Altogether, the qPCR data further 
highlights that in Mtb exogenous heme is metabolized differently than endogenously synthesized heme.

Iron availability regulates $\mathrm{ChdC}$ expression and heme uptake in $M$. tuberculosis. Since ChdC was found to be a negative regulator of heme uptake and utilization, we sought to model conditions experienced by Mtb during infection that may increase or decrease ChdC expression and in turn alter heme uptake and/or utilization. Given that heme can be used as an iron source under iron depleted conditions,(22) iron availability is necessary for heme synthesis, and ablating chdC increased heme uptake, we examined whether iron availability affected ChdC protein levels and heme uptake. Through immunoblotting experiments using a polyclonal anti-Mtb ChdC antibody, we determined that ChdC expression was decreased under low iron conditions in minimal media (MM) supplemented with $5 \mu \mathrm{M}$ Fe (Fig. 2 e,f) compared to MM with $150 \mu \mathrm{M}$ Fe.

To determine whether iron availability affected heme uptake in Mtb, we measured uptake of the heme analog ZnMP in Fe limited (5 $\mu \mathrm{M} \mathrm{Fe)}$ and Fe replete (150 $\mu \mathrm{M}$ Fe) conditions. WT Mtb cells grown in MM supplemented with $150 \mu \mathrm{M}$ Fe had ZnMP uptake that was $\sim 30 \%$ of WT Mtb grown in $5 \mu \mathrm{M}$ Fe (Fig. 2 g,h).

Finally, we assessed the ability of WT Mtb to use exogenous heme for growth in iron limiting conditions that correspond to decreased ChdC expression ( $\mathrm{MM}+5 \mu \mathrm{M} \mathrm{Fe}$ ). The addition of $12.5 \mu \mathrm{M}$ heme increased WT Mtb growth in MM supplemented with $5 \mu \mathrm{M}$ Fe and to the same level as cells treated with $150 \mu \mathrm{M}$ Fe (Fig. 2i). Therefore, iron dependent ChdC expression negatively correlated with both an increase in the uptake of heme analog ZnMP and the effect of heme supplementation on WT Mtb growth in MM. 
Exogenous heme decreases porphyrin synthesis in M. tuberculosis. Heme synthesis is regulated by intracellular heme levels in some bacteria (11) and the decrease of $g$ trR transcript levels in WT Mtb with heme treatment (Fig. 2d) suggested that this regulation may occur in Mtb as well. To better determine if exogenous heme provides feedback to regulate heme synthesis in mycobacteria, we measured fluorescent porphyrins as a proxy for flux through the heme biosynthetic pathway. The results showed that exogenous heme decreased porphyrin levels in Mtb similar to inhibition of heme synthesis by succinylacetone, suggesting that heme does regulate heme synthesis early in the biosynthetic pathway. (Supplemental Appendix and Fig. S8a-h) Additionally, culturing WT Mtb in iron deplete MM (5 $\mu \mathrm{M} \mathrm{Fe})$ did not reduce porphyrin or heme levels compared to iron replete MM (150 $\mu \mathrm{M} \mathrm{Fe)} \mathrm{(} \mathrm{Fig.} \mathrm{S8} \mathrm{i,j).} \mathrm{Taken}$ together with the RT-qPCR results (Fig. 2d), we propose that heme feeds back to regulate the synthesis of early porphyrin intermediates and that this feedback likely requires gtrR.

ChdC regulates a reductive heme uptake pathway in $M$. tuberculosis. In order to determine biochemical features of ChdC-regulated heme uptake, and ultimately its mechanism of action, we assessed if ChdC inhibited heme uptake in an oxidation-state dependent manner. Towards this end, we first assessed if Mtb exhibited a preference to internalize divalent zinc(II)protoporphyrin IX (ZnPP) or trivalent gallium(III)protoporphyrin IX (GaPP), which are redox inactive fluorescent heme analogs that serve as mimics of ferrous $\left(\mathrm{Fe}^{2+}\right)$ or ferric $\left(\mathrm{Fe}^{3+}\right)$ heme, respectively, due to similar metal oxidation numbers and ionic radii. (33-37) In standard growth conditions of 7H9 + ADS, ZnPP accumulated in WT Mtb to a far greater extent (nearly 3-fold in 48 hours) than GaPP (Fig. 3a). We next compared GaPP vs. ZnPP uptake in WT, $\Delta$ gtrR and $\Delta c h d C$ Mtb in 7H9+ADS after 
48 hours. In $\triangle$ chdC Mtb, the ratio of GaPP to ZnPP uptake increased 3-fold and approached 1 (Fig. 3b), which indicated that $\Delta c h d C$ cells lost the ability to discriminate between the $\mathrm{Fe}^{3+}$-heme mimic GaPP and $\mathrm{Fe}^{2+}$-heme mimic $\mathrm{ZnPP}$.

This observed preference for transporting ZnPP over GaPP in WT Mtb led us to develop a modified pyridine hemochromagen assay (MPH, see Supplemental methods) for determining the concentration of ferrous heme in Mtb cells. $(38,39)$ WT, $\Delta g t r R$ and $\triangle c h d C$ Mtb cells treated with $25 \mu \mathrm{M}$ heme for 7 days in $7 \mathrm{H} 9+$ ADS with aeration, all had $\sim 35-50 \%$ ferrous heme in whole cells lysed in pyridine hemochromagen solution (PHS, see Supplemental methods) (Fig. S9a). The results in $\Delta g t r R$ and $\Delta$ chdC Mtb, which cannot synthesize heme, implied that ferric exogenous heme was reduced either during uptake, in the cytosol, or both (Fig. S9a). To assess whether Mtb could reduce extracellular heme, WT, $\Delta g t r R$ and $\Delta c h d C$ Mtb cells were incubated with $5 \mu \mathrm{M}$ ferric heme in PBS in an anaerobic chamber for 24 hours. Cells were pelleted and resuspended in PHS for 1-2 minutes and then pelleted again. Heme remaining in the cell-free PHS supernatant was measured by the MPH assay and is referred to as cellsurface associated heme, given that it was likely associated with the outer cell surface and/or possibly the periplasm. The MPH assay revealed that $\sim 1 \%$ of cell surface associated heme was reduced in WT Mtb (Fig. 3c). Most interestingly, $\triangle$ chdC cells exhibited a nearly 3-fold greater surface heme reductase activity than WT cells (Fig. 3c). $\Delta g t r R$ Mtb cells exhibited similar surface heme reductase activity to WT cells, suggesting that extracellular heme reduction is inhibited by ChdC and not general heme synthesis. In the presence of atmospheric oxygen $\left(\mathrm{O}_{2}\right)$, we could not observe heme reduction at the cell surface, indicating that $\mathrm{O}_{2}$ could re-oxidize reduced heme. Disrupted cell lysates also contained a ferric heme reducing activity (Fig. S9b-d) that was lost upon boiling (Fig. 
S9e), indicating that the heme reductase activity likely arises from an enzyme (Fig. S9b,c). However, interestingly, unlike in intact cells, cell lysates from $\Delta c h d C$ cells did not have an increase in heme reducing activity, suggesting that upon lysis, ChdC was decoupled from regulating heme reductase activity (Fig. $\mathbf{S 9} \mathbf{d , f})$.

The identification of heme reductase activity in Mtb led us to determine if exogenously supplied ferric heme is more bioavailable to cells in the absence of $\mathrm{O}_{2}$. Towards this end, we measured LH in WT Mtb expressing HS1-M7A after 24 hours in PBS supplemented with $5 \mu \mathrm{M}$ exogenous heme in air versus an anaerobic chamber (Fig. 3d). Upon normalizing the sensor signal to the heme invariant HS1-M7A, H102A to account for heme-independent changes in fluorescence signal, which were minimal, we found heme bioavailability was significantly elevated in the absence of $\mathrm{O}_{2}$, consistent with a pathway for reductive heme uptake. Altogether, our results suggest that ChdC regulates heme import by inhibiting a reductive heme uptake pathway.

Role of endogenous heme synthesis in macrophage infection. Given that Mtb may reside in macrophages where iron availability is limited to the pathogen,(40) we sought to determine if ChdC plays a role in Mtb survival and replication in the macrophage. Towards this end, we employed an in vitro assay of intracellular infection using RAW 264.7 macrophages in order to determine differences in metabolic activity between $\Delta g t r R$ and $\triangle c h d C$ Mtb in response to macrophage infection (41). We measured the reduction of the tetrazolium dye MTT, which forms a purple formazan, as a readout of metabolic activity and viability in Mtb $(42,43)$. MTT-reducing activity of WT and heme depleted $\Delta$ gtrR and $\triangle$ chdC Mtb was measured before and 24-hours after RAW macrophage infection. Prior to infection, WT and heme depleted $\Delta g t r R$ and $\Delta c h d C$ Mtb 
had similar metabolic activity (Fig. 4a). However, in Mtb cells isolated from macrophages after infection, both $\Delta g t r R$ and $\Delta c h d C$ Mtb had a $>30 \%$ decrease in metabolic activity compared to WT Mtb (Fig. 4b). This highlighted the importance of de novo heme synthesis during macrophage infection. There was also a difference in metabolic activity between the two heme auxotrophic strains, as $\triangle$ chdC Mtb cells had greater MTTreducing activity than $\Delta g t r R$ Mtb cells. We suggest that this is due to the ability of $\Delta c h d C$ Mtb to more efficiently acquire and utilize exogenous heme than $\Delta g t r R$ Mtb. However, we cannot rule out a role for heme biosynthetic pathway intermediates in macrophage survival. Altogether, our results indicated that both heme biosynthesis and uptake were important for survival in macrophages.

\section{Discussion}

Heme is both an essential cofactor and a source of nutritional iron for the human pathogen Mtb (22). The importance of heme in Mtb physiology is underscored by the fact that there are at least two independent routes for heme acquisition, and heme can be synthesized de novo by $\operatorname{Mtb}(8,10)$. To balance the nutritional and metabolic demands for iron and heme, while also mitigating the potential for heme toxicity, Mtb must tightly coordinate heme uptake, synthesis and utilization and integrate these processes with iron availability. Herein, our work identifies a previously uncharacterized mechanism in which the terminal heme biosynthetic enzyme, coproheme decarboxylase $(\mathrm{ChdC})$, negatively regulates heme uptake and utilization through a reductive heme uptake pathway (Fig. 4c). Iron limitation down-regulates ChdC and increases heme import and bioavailability. (Fig. 2 e-i). We propose that such a mechanism may serve to protect cells from heme toxicity while trying to meet the nutritional demand for iron via 
heme scavenging and uptake from the host. These findings have a number of implications for iron and heme homeostasis in Mtb at the host-pathogen axis.

Heme is a cofactor necessary for Mtb virulence as heme dependent proteins such as KatG, $(30,44)$ cytochrome P450s,(45) and the Dos two component system,(46) are required for the survival and virulence of Mtb in infection models $(23,30)$. However, the relative contributions of de novo synthesized and exogenously scavenged heme towards labile bioactive heme pools and protein hemylation were unknown. Herein, we found that under conditions in which endogenously synthesized and exogenously supplied heme contributed equally to the total cellular heme concentration (Fig. 1b), exogenous heme was poorly utilized by mycobacteria and was inefficient at rescuing the growth of a heme deficient $\Delta g t r R$ strain (Figs. 1a and 2a), contributing to the LH pool (Fig. 1c) and activating the heme enzyme KatG (Fig. 1d). Additionally, using an Mtbmacrophage infection assay we found that survival of the heme synthetic mutants, $\Delta g t r R$ and $\triangle c h d C$, were impaired in infected macrophages compared to WT Mtb (Fig. 4b). Our findings that de novo synthesized heme is important for Mtb survival was also supported by prior studies indicating that many enzymes of the Mtb heme synthetic pathway were upregulated in Tb patient lungs compared with in vitro growth conditions.(47) We also found that LH levels were a good indicator of the bioactivity of subcellular heme pools in mycobacteria, including for activation of KatG, which is necessary for survival of Mtb in the macrophage $(23,30)$ and expressed in clinical isolates of Tb patients.(44)

One potential explanation for the apparent poor bioavailability of exogenously supplied heme is that it is degraded. However, we counterintuitively found that the transcript for the heme degrading heme oxygenase, MhuD, is repressed by heme supplementation and induced under heme deficient conditions (Fig. 2d), suggesting that 
heme degradation did not account for the differences in bioavailability between exogenously transported and endogenously made heme. Nonetheless, we cannot rule out this possibility as we have been unable to measure mycobilin, a product of heme degradation in mycobacteria, from cell extracts and therefore assess the extent to which heme is degraded in vivo.

An alternative explanation is that Mtb may regulate the trafficking and bioavailability of endogenously synthesized and exogenously scavenged heme differently due to differences in oxidation state and its concomitant effects on subcellular localization. Heme synthesized via ChdC is ferric, as ferric iron in coproheme is required for decarboxylation to heme.(48, 49) However, since the major mycobacterial redox buffer is mycothiol, which sets the cytosolic redox poise to highly reducing values, between -320 and $-220 \mathrm{mV}$ vs. the normal hydrogen electrode (NHE),(50) newly synthesized ferric heme may be rapidly reduced to ferrous heme, assuming its potential is similar to monomeric aqueous heme, $(-50 \mathrm{mV}$ vs. NHE), $(1,51)$ and labile heme equilibrates with the mycothiol redox buffer. Thus, one may reasonably expect that cytosolic heme is largely in its reduced ferrous state. In contrast, exogenous extracellular heme, which is in its oxidized ferric state, may gain access to the periplasmic space, which accounts for its contribution to "total" heme levels, but cannot be transported across the plasma membrane into the cytosol unless it is reduced by the ferric heme reductase system, thereby accounting for its comparatively low bioavailability. Ablation of ChdC, which otherwise serves to repress ferric heme reductase activity, results in elevated exogenous heme reduction (Fig. 3c) and uptake (Figs. $\mathbf{2 b}$ and $\mathbf{2 c}$ ), which would explain its increased availability in $\triangle c h d C$ cells. Indeed, our findings that WT Mtb took up more ferrous heme analog, ZnPP, than ferric heme analog, GaPP, (Fig. 3 a,b), 
and that ferric exogenous heme was more bioavailable in the absence of $\mathrm{O}_{2}$ (Fig. $\mathbf{3 d}$ ) support the proposal that exogenously acquired heme is trafficked more efficiently into the cytosol upon reduction into its ferrous state. Therefore, in sum, heme iron oxidation state may be a key determinant that leads to the differential trafficking and bioavailability of exogenously scavenged and endogenously synthesized heme.

What is the nature of the ferric heme reductase and transport system? It likely requires electrons from $\mathrm{NADH}$ or $\mathrm{NADPH}$ and utilizes a flavin to ultimately reduce exogenous $\mathrm{Fe}^{3+}$-heme. Prior studies have implicated electron transfer flavoproteins, Fix $A B$, as being required for utilization of heme as an iron source,(10) raising the intriguing possibility that FixAB are components of the ferric heme reductase system. The reductase system is likely present in the plasma membrane, where it would have access to cytosolically produced $\mathrm{NAD}(\mathrm{P}) \mathrm{H}$. As such, we propose a model in which extracellular $\mathrm{Fe}^{3+}$-heme partitions into the periplasmic space, via outer membrane proteins such as porins or heme transporters, e.g. PPE62, or possibly diffusion through the cell envelope and outer-membrane, where it can be reduced by the ferric heme reductase system and transported across the plasma membrane into the cytosol.

Interestingly, deletion of ChdC disrupts the ability of Mtb cells to selectively uptake the ferrous heme mimic, ZnPP, over ferric heme mimic, GaPP (Fig. 3b). Given that both $\mathrm{ZnPP}$ and GaPP are redox inactive, it would suggest that ChdC is also required for the unknown heme transport system to confer selectivity for ferrous heme uptake, in addition to the ferric heme reductase activity. Taken together, we propose that when ChdC expression is increased in iron replete conditions, heme synthesis is active and heme uptake is repressed due to the inhibition of the ferric heme reductase and enhanced selectivity in transport of ferrous heme, thereby limiting uptake of exogenous 
oxidized heme. When ChdC expression is low, heme synthesis is diminished and heme uptake is elevated due to the de-repression of the ferric heme reductase and enhanced transport of both ferrous and ferric heme. Coupling heme reduction to ferrous selective transport using a heme biosynthetic enzyme may ensure Mtb does not inappropriately accumulate toxic levels of heme.

Exactly how heme reduction is coupled to its transport and the mechanisms underlying ChdC-mediated inhibition of heme reductase activity and selectivity in ferrous heme uptake remains to be determined. Since the ChdC-dependent heme reductase activity is lost in disrupted cell lysates (Fig. S9b), there may be key protein-protein interactions and a defined molecular architecture of ChdC and the heme reductase and transport systems akin to the heme biosynthetic metabolon discovered in the mitochondrial inner-membrane of eukaryotes.(52-54) Alternatively, since ablation of ChdC results in the inability to sense heme as evidenced by the lack of a transcriptional signature consistent with heme starvation in $\Delta c h d C$ cells (Fig. $2 \mathbf{d}$ ), ChdC may act as an intracellular sensor of heme that regulates the expression of additional factor(s) that control heme reductase activity and uptake.

Heme uptake pathways are important for Mtb survival in macrophage and mouse infection models. $(8,55)$ While iron levels are considered to be sufficient to support Mtb growth within the macrophage (56) or outer layers of the granuloma,(57) the caseous center of the granuloma is predicted to be severely iron limiting.(57) Our findings that iron limitation down-regulates $\mathrm{ChdC}$ protein level and increases heme import and bioavailability (Fig. 2 g-i) suggest that ChdC-regulated heme transport may be an important component of the iron starvation response in Mtb and is required for scavenging heme-iron from the host. While extracellular iron levels regulate the 
transcription of genes encoding other enzymes in the CPD pathway, including glutamate-semialdehyde amino mutase, GsaM, (also listed as HemL) and $\mathrm{CpfC}$ (also listed as HemZ),(57) the changes in chdC expression from multiple transcriptomic studies are distinct from other heme synthetic enzymes, further highlighting the unique role of $\mathrm{ChdC}$ in coordinating heme synthesis and uptake in response to iron availability. Further studies are needed to clarify the relationship between iron availability within the host and ChdC protein expression.

Iron regulation of ChdC likely occurs at the post-translational level given that prior transcriptomic studies have not shown that ChdC mRNA levels vary in response to changes in iron levels. $(57,58) \mathrm{ChdC}$ is part of a subset of Mtb proteins (including only one other heme synthesis enzyme, GsaM), degraded via the CLP protease.(59) The CLP protease degrades proteins under non-stress conditions, suggesting ChdC is actively turned-over. Since ChdC catalytic activity is required to suppress heme uptake and utilization, it is tempting to speculate that iron regulation of ChdC expression occurs through a mechanism that involves differential turn-over of heme or coproheme bound and unbound forms of ChdC via the CLP protease.

The specific molecular mechanisms underlying ChdC-mediated regulation of heme uptake, bioavailability and utilization also remains to be determined. While we have shown that ChdC regulates a reductive heme uptake pathway, we were unable to transcriptionally identify known heme transport genes involved in ChdC regulated heme uptake (Fig. 2d). We predict that as-yet-to-be determined ChdC protein-protein interactions are involved in regulating factors that control heme reduction, uptake and bioavailability. For example, in Staphylococcus aureus, ChdC interacts with coproporphyrin ferrochelatase $(\mathrm{CpfC})$ and IsdG, one of two heme degrading 
enzymes. $(60,61)$ Given that catalytically active $C h d C$ is required to suppress heme uptake and bioavailability (Fig. S4), we propose that transfer of heme from ChdC to a downstream target inhibits heme reduction, uptake and/or bioavailability. For instance, protein-protein interactions have been identified that transport newly synthesized heme from the terminal enzyme of the PPD branch, ferrochelatase, to downstream hemoproteins.(62) Alternatively, it is possible that heme-bound ChdC has a distinct interaction network from heme-free ChdC to control heme uptake. Identification of the ChdC interactome will clarify how $\mathrm{ChdC}$ regulates heme uptake and utilization.

Post translational modifications (PTMs) of ChdC may be critical for regulating its expression and/or interactions with factors that regulate heme uptake and bioavailability. Mtb ChdC is both succinylated (63) and pupylated. (64) Both succinylation and pupylation occur at surface exposed lysines and could inhibit protein-protein interactions, or in the case of pupylation, also mark the protein for degradation.(64) Interestingly, pupylation has already been shown to be an iron dependent regulator of ferritin in Corynebacterium glutamicum.(65) In C. glutamicum, ChdC is also pupylated, however, no pup-dependent proteasomal machinery exists.(66) Moreover, current studies do not support pupylation dependent degradation of $\mathrm{ChdC}$, suggesting pupylation of $\mathrm{ChdC}$ may play a regulatory role.(67) Further studies are warranted to clarify how ChdC PTMs affect expression, activity, and regulation of heme uptake and bioavailability.

One surprising finding from our work was the level of free porphyrins in WT Mtb (Fig. S8). The buildup of heme intermediates is considered disadvantageous due to the inherent cytotoxicity of porphyrins. In Msm, the build-up of porphyrins in cultures during the transition to dormancy increases the susceptibility of Msm to photoinactivation. (68) As has been proposed for the treatment of other bacteria(69) and cancers, the 
exploitation of light enhanced porphyrin toxicity may underly the basis of future anti-Mtb therapies. (70)

Altogether, our studies are the first to demonstrate that the regulation of heme synthesis, uptake and bioavailability in mycobacteria requires the heme synthetic enzyme, ChdC, whose expression is both iron and heme regulated, and which controls a reductive heme uptake pathway (Fig. 4c). A detailed knowledge of how ChdC coordinates heme uptake and utilization and the specific biological contexts in which it does so will be essential for understanding how Mtb adapts to varying availability of iron, heme and oxygen during infection.

\section{Materials and Methods}

All materials, including reagents, cell lines, culture conditions, and methods are described in detail in the Supplemental Material and Methods. Mtb strains used in this work are avirulent and in the $\mathrm{mc}^{2} 6230(\mathrm{H} 37 \mathrm{Rv} \Delta r d 1 \triangle p a n C D)$ background. Msm strains are in the $\mathrm{mc}^{2} 155$ background. Generation of knockout strains and sensor incorporation in mycobacteria are also described in Supplemental Material and Methods. All experimental methods including total heme assay,(71) labile heme,(16) sensor calibration,(16) porphyrin assay,(71) catalase-peroxidase activity assay, $(24,25)$ fluorescent porphyrin assays, and ferrous heme measurements are also described in Supplemental Material and Methods in the Supplemental Appendix.

Acknowledgment: We thank Prof. William R. Jacobs, Jr. (Albert Einstein College of Medicine) for sharing $M$. smegmatis $\mathrm{mc}^{2} 155$, M. tuberculosis $\mathrm{mc}^{2} 6230$, and the specialized transduction system and base plasmids used in this work. We thank Prof. Daniel Kornitzer for helpful discussions regarding heme reductase activity. We wish to 
acknowledge the core facilities at the Parker $\mathrm{H}$. Petit Institute for Bioengineering and Bioscience at the Georgia Institute of Technology for the use of their shared equipment, services and expertise.

\section{Funding}

This research was supported by US National Institutes of Health grants ES025661 (to AR) and Al137338 (to MN and AR), a US-Israel Binational Science Foundation grant 2017228 (to AR and Daniel Kornitzer), a US National Science Foundation grant 1552791 (to AR), and the Stony Wold-Herbert Fund (to PJ).

\section{References}

1. Donegan RK, Moore CM, Hanna DA, \& Reddi AR (2019) Handling heme: The mechanisms underlying the movement of heme within and between cells. Free Radical Biology and Medicine 133:88-100.

2. McLean KJ \& Munro AW (2017) Drug targeting of heme proteins in Mycobacterium tuberculosis. Drug Discovery Today 22(3):566-575.

3. Andrews NC (1999) Disorders of iron metabolism. New England Journal of Medicine 341(26):1986-1995.

4. Reddi AR \& Hamza I (2016) Heme mobilization in animals: a Metallolipid's journey. Accounts of chemical research 49(6):1104-1110.

5. Hanna DA, Martinez-Guzman O, \& Reddi AR (2017) Heme Gazing: Illuminating Eukaryotic Heme Trafficking, Dynamics, and Signaling with Fluorescent Heme Sensors. Biochemistry 56(13):1815-1823.

6. Owens CP, Chim N, \& Goulding CW (2013) Insights on how the Mycobacterium tuberculosis heme uptake pathway can be used as a drug target. Future medicinal chemistry 5(12):10.4155/fmc.4113.4109.

7. Mitra A, Speer A, Lin K, Ehrt S, \& Niederweis M (2017) PPE surface proteins are required for heme utilization by Mycobacterium tuberculosis. mBio 8:e01720-01716.

8. Mitra A, Ko Y-H, Cingolani G, \& Niederweis M (2019) Heme and hemoglobin utilization by Mycobacterium tuberculosis. Nature Communications 10(1):4260. 
9. Tullius MV, et al. (2011) Discovery and characterization of a unique mycobacterial heme acquisition system. Proceedings of the National Academy of Sciences of the United States of America 108(12):5051-5056.

10. Zhang L, et al. (2020) Comprehensive analysis of iron utilization by Mycobacterium tuberculosis. PLoS pathogens 16(2):e1008337.

11. Dailey HA, et al. (2017) Prokaryotic Heme Biosynthesis: Multiple Pathways to a Common Essential Product. Microbiology and Molecular Biology Reviews 81(1):e00048-00016.

12. Celis Al \& DuBois JL (2019) Making and breaking heme. Current Opinion in Structural Biology 59:19-28.

13. Dailey HA, Gerdes S, Dailey TA, Burch JS, \& Phillips JD (2015) Noncanonical coproporphyrin-dependent bacterial heme biosynthesis pathway that does not use protoporphyrin. Proceedings of the National Academy of Sciences 112(7):2210-2215.

14. Dailey TA, et al. (2010) Discovery and characterization of HemQ an essential heme biosynthetic pathway component. Journal of Biological Chemistry 285(34):25978-25986.

15. Protchenko O, et al. (2008) Role of PUG1 in inducible porphyrin and heme transport in Saccharomyces cerevisiae. Eukaryotic cell 7(5):859-871.

16. Hanna DA, et al. (2016) Heme dynamics and trafficking factors revealed by genetically encoded fluorescent heme sensors. Proceedings of the National Academy of Sciences 113(27):7539-7544.

17. Hanna DA, et al. (2018) Heme bioavailability and signaling in response to stress in yeast cells. Journal of Biological Chemistry 293(32):12378-12393.

18. Sweeny EA, et al. (2018) Glyceraldehyde-3-phosphate dehydrogenase is a chaperone that allocates labile heme in cells. Journal of Biological Chemistry 293(37):14557-14568.

19. Martinez-Guzman O, et al. (2020) Mitochondrial-nuclear heme trafficking in budding yeast is regulated by GTPases that control mitochondrial dynamics and ER contact sites. Journal of Cell Science 133(10):jcs237917.

20. Dai Y, Sweeny EA, Schlanger S, Ghosh A, \& Stuehr DJ (2020) GAPDH delivers heme to soluble guanylyl cyclase. Journal of Biological Chemistry 295(24):8145-8154.

21. Lindblad B, Lindstedt S, \& Steen G (1977) On the enzymic defects in hereditary tyrosinemia. Proceedings of the National Academy of Sciences 74(10):4641-4645.

22. Jones CM \& Niederweis M (2011) Mycobacterium tuberculosis can utilize heme as an iron source. Journal of bacteriology 193(7):1767-1770.

23. Forrellad MA, et al. (2013) Virulence factors of the Mycobacterium tuberculosis complex. Virulence 4(1):3-66.

24. Wayne LG \& Diaz GA (1986) A double staining method for differentiating between two classes of mycobacterial catalase in polyacrylamide electrophoresis gels. Analytical biochemistry 157(1):89-92.

25. Woodbury W, Spencer A, \& Stahmann M (1971) An improved procedure using ferricyanide for detecting catalase isozymes. Analytical biochemistry 44(1):301-305.

26. Chim N, Iniguez A, Nguyen TQ, \& Goulding CW (2010) Unusual diheme conformation of the heme-degrading protein from Mycobacterium tuberculosis. Journal of molecular biology 395(3):595-608.

27. Ahmed FH, et al. (2016) Rv2074 is a novel F420H2-dependent biliverdin reductase in Mycobacterium tuberculosis. Protein Science 25(9):1692-1709. 
28. Tullius MV, Nava S, \& Horwitz MA (2019) PPE37 Is Essential for Mycobacterium tuberculosis Heme-Iron Acquisition (HIA), and a Defective PPE37 in Mycobacterium bovis BCG Prevents HIA. Infection and Immunity 87(2):e00540-00518.

29. Khare G, Nangpal P, \& Tyagi AK (2017) Differential roles of iron storage proteins in maintaining the iron homeostasis in Mycobacterium tuberculosis. PLoS One 12(1):e0169545.

30. Ng VH, Cox JS, Sousa AO, MacMicking JD, \& McKinney JD (2004) Role of KatG catalase-peroxidase in mycobacterial pathogenesis: countering the phagocyte oxidative burst. Molecular microbiology 52(5):1291-1302.

31. Rodriguez GM, Voskuil MI, Gold B, Schoolnik GK, \& Smith I (2002) ideR, an essential gene in Mycobacterium tuberculosis: role of IdeR in iron-dependent gene expression, iron metabolism, and oxidative stress response. Infection and immunity 70(7):33713381.

32. Gold B, Rodriguez GM, Marras SA, Pentecost M, \& Smith I (2001) The Mycobacterium tuberculosis IdeR is a dual functional regulator that controls transcription of genes involved in iron acquisition, iron storage and survival in macrophages. Molecular microbiology 42(3):851-865.

33. Tangar A, et al. (2019) Utility of fluorescent heme analogue ZnPPIX to monitor conformational heterogeneity in vertebrate hexa-coordinated globins. Metallomics 11(5):906-913.

34. Xu S \& Kaltashov IA (2016) Evaluation of Gallium as a Tracer of Exogenous HemoglobinHaptoglobin Complexes for Targeted Drug Delivery Applications. Journal of The American Society for Mass Spectrometry 27(12):2025-2032.

35. Hijazi S, Visca P, \& Frangipani E (2017) Gallium-protoporphyrin IX inhibits Pseudomonas aeruginosa growth by targeting cytochromes. Frontiers in cellular and infection microbiology 7:12.

36. Chitambar CR (2016) Gallium and its competing roles with iron in biological systems. Biochimica et Biophysica Acta (BBA)-Molecular Cell Research 1863(8):2044-2053.

37. Deraz N \& Alarifi A (2009) Synthesis and characterization of pure and Li2O doped ZnFe2O4 nanoparticles via glycine assisted route. Polyhedron 28(18):4122-4130.

38. Berry EA \& Trumpower BL (1987) Simultaneous determination of hemes $a, b$, and c from pyridine hemochrome spectra. Analytical biochemistry 161(1):1-15.

39. Barr I \& Guo F (2015) Pyridine Hemochromagen Assay for Determining the Concentration of Heme in Purified Protein Solutions. Bio Protoc 5(18):e1594.

40. Ganz T (2009) Iron in innate immunity: starve the invaders. Current Opinion in Immunology 21(1):63-67.

41. Mouton JM, et al. (2019) Comprehensive Characterization of the Attenuated Double Auxotroph Mycobacterium tuberculosis $\triangle$ leuD $\Delta$ panCD as an Alternative to H37Rv. Front Microbiol 10:1922-1922.

42. Hundie GB, Woldemeskel D, \& Gessesse A (2016) Evaluation of direct colorimetric MTT assay for rapid detection of rifampicin and isoniazid resistance in mycobacterium tuberculosis. PLoS One 11(12):e0169188.

43. Tada H, Shiho O, Kuroshima K-i, Koyama M, \& Tsukamoto K (1986) An improved colorimetric assay for interleukin 2. Journal of immunological methods 93(2):157-165. 
44. Singhal N, Sharma P, Kumar M, Joshi B, \& Bisht D (2012) Analysis of intracellular expressed proteins of Mycobacterium tuberculosis clinical isolates. Proteome Science 10(1):14.

45. Ouellet H, Johnston JB, \& Ortiz de Montellano PR (2010) The Mycobacterium tuberculosis cytochrome P450 system. Archives of biochemistry and biophysics 493(1):82-95.

46. Gautam US, et al. (2015) DosS Is required for the complete virulence of mycobacterium tuberculosis in mice with classical granulomatous lesions. American journal of respiratory cell and molecular biology 52(6):708-716.

47. Rachman $\mathrm{H}$, et al. (2006) Unique transcriptome signature of Mycobacterium tuberculosis in pulmonary tuberculosis. Infection and immunity 74(2):1233-1242.

48. Celis Al, et al. (2017) Structure-based mechanism for oxidative decarboxylation reactions mediated by amino acids and heme propionates in coproheme decarboxylase (HemQ). Journal of the American Chemical Society 139(5):1900-1911.

49. Celis Al, et al. (2015) Unusual peroxide-dependent, heme-transforming reaction catalyzed by HemQ. Biochemistry 54(26):4022-4032.

50. Mehta M \& Singh A (2019) Mycobacterium tuberculosis WhiB3 maintains redox homeostasis and survival in response to reactive oxygen and nitrogen species. Free Radical Biology and Medicine 131:50-58.

51. Conant JB \& Tongberg C (1930) The oxidation-reduction potentials of hemin and related substances: I. The potentials of various hemins and hematins in the absence and presence of pyridine. Journal of Biological Chemistry 86(2):733-741.

52. Swenson SA, et al. (2020) From synthesis to utilization: the ins and outs of mitochondrial heme. Cells 9(3):579.

53. Piel III RB, Dailey Jr HA, \& Medlock AE (2019) The mitochondrial heme metabolon: Insights into the complex (ity) of heme synthesis and distribution. Molecular genetics and metabolism 128(3):198-203.

54. Medlock AE, et al. (2015) Identification of the mitochondrial heme metabolism complex. PloS one 10(8):e0135896.

55. Flores-Valdez MA, Morris RP, Laval F, Daffé M, \& Schoolnik GK (2009) Mycobacterium tuberculosis modulates its cell surface via an oligopeptide permease (Opp) transport system. The FASEB Journal 23(12):4091-4104.

56. Boelaert JR, Vandecasteele SJ, Appelberg R, \& Gordeuk VR (2007) The effect of the host's iron status on tuberculosis. The Journal of infectious diseases 195(12):1745-1753.

57. Kurthkoti K, et al. (2017) The Capacity of Mycobacterium tuberculosis To Survive Iron Starvation Might Enable It To Persist in Iron-Deprived Microenvironments of Human Granulomas. mBio 8(4):e01092-01017.

58. Bacon J, et al. (2007) Lipid composition and transcriptional response of Mycobacterium tuberculosis grown under iron-limitation in continuous culture: identification of a novel wax ester. Microbiology (Reading, England) 153(Pt 5):1435.

59. Lunge A, Gupta R, Choudhary E, \& Agarwal N (2020) The unfoldase ClpC1 of Mycobacterium tuberculosis regulates the expression of a distinct subset of proteins having intrinsically disordered termini. Journal of Biological Chemistry:jbc. RA120. 013456. 
60. Videira MA, et al. (2018) Staphylococcus aureus haem biosynthesis and acquisition pathways are linked through haem monooxygenase IsdG. Molecular microbiology 109(3):385-400.

61. Celis Al, Choby JE, Kentro J, Skaar EP, \& DuBois JL (2019) Control of metabolite flux during the final steps of heme $b$ biosynthesis in Gram positive bacteria. Biochemistry.

62. Piel RB, et al. (2016) A Novel Role for Progesterone Receptor Membrane Component 1 (PGRMC1): A Partner and Regulator of Ferrochelatase. Biochemistry 55(37):5204-5217.

63. Yang $M$, et al. (2015) Succinylome analysis reveals the involvement of lysine succinylation in metabolism in pathogenic Mycobacterium tuberculosis. Mol Cell Proteomics 14(4):796-811.

64. Festa RA, et al. (2010) Prokaryotic ubiquitin-like protein (Pup) proteome of Mycobacterium tuberculosis [corrected]. PloS one 5(1):e8589-e8589.

65. Küberl A, Polen T, \& Bott M (2016) The pupylation machinery is involved in iron homeostasis by targeting the iron storage protein ferritin. Proceedings of the National Academy of Sciences 113(17):4806-4811.

66. Küberl A, et al. (2014) Pupylated proteins in Corynebacterium glutamicum revealed by MudPIT analysis. Proteomics 14(12):1531-1542.

67. Fascellaro G, et al. (2016) Comprehensive Proteomic Analysis of Nitrogen-Starved Mycobacterium smegmatis $\Delta$ pup Reveals the Impact of Pupylation on Nitrogen Stress Response. Journal of proteome research 15(8):2812-2825.

68. Shleeva MO, et al. (2019) Photoinactivation of dormant Mycobacterium smegmatis due to its endogenous porphyrins. Applied microbiology and biotechnology 103(23-24):96879695.

69. Malik Z, Hanania J, \& Nitzan Y (1990) New trends in photobiology bactericidal effects of photoactivated porphyrins-An alternative approach to antimicrobial drugs. Journal of Photochemistry and Photobiology B: Biology 5(3-4):281-293.

70. Hirakawa K, et al. (2018) Photosensitized protein-damaging activity, cytotoxicity, and antitumor effects of $\mathrm{P}(\mathrm{V})$ porphyrins using long-wavelength visible light through electron transfer. Chemical research in toxicology 31(5):371-379.

71. Sassa S (1976) Sequential induction of heme pathway enzymes during erythroid differentiation of mouse Friend leukemia virus-infected cells. The Journal of Experimental Medicine 143(2):305-315. 
Figures and Tables

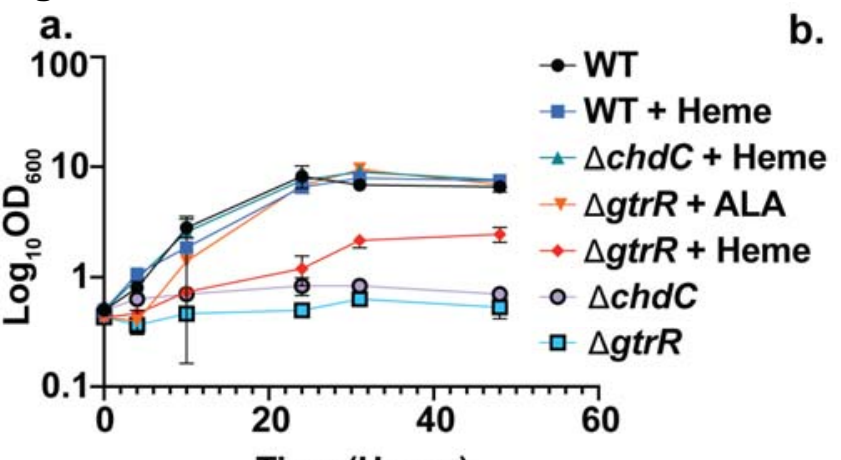

c.

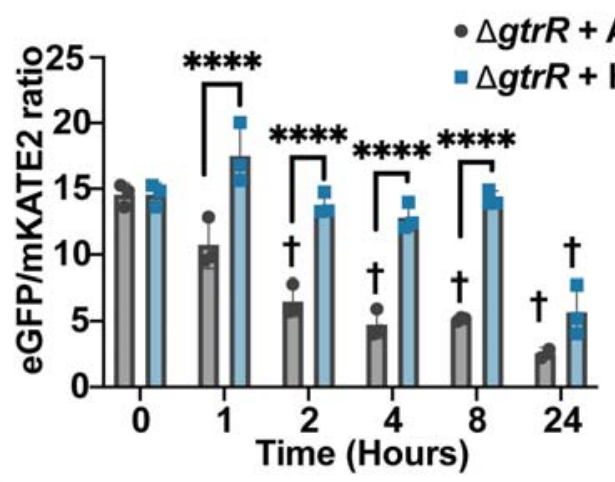

e.

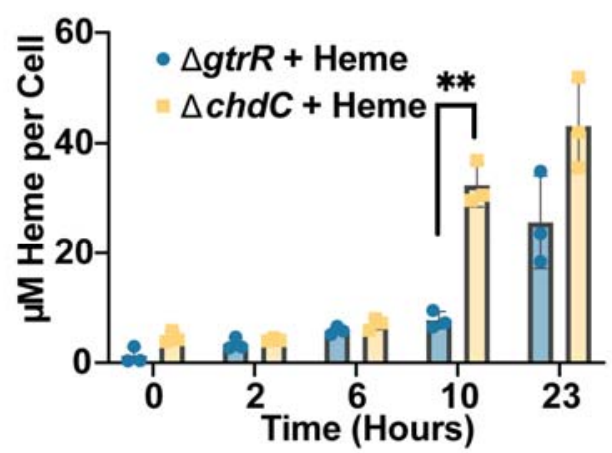

g.

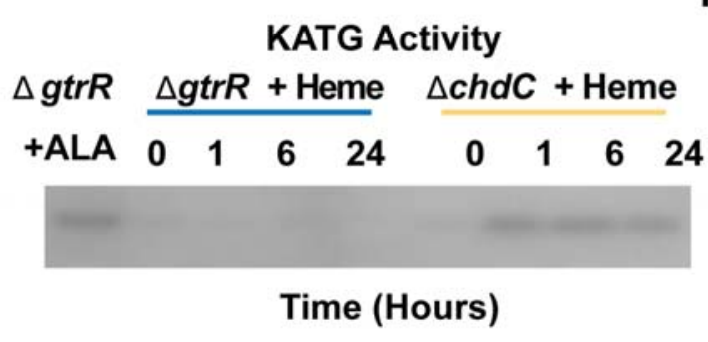

b.

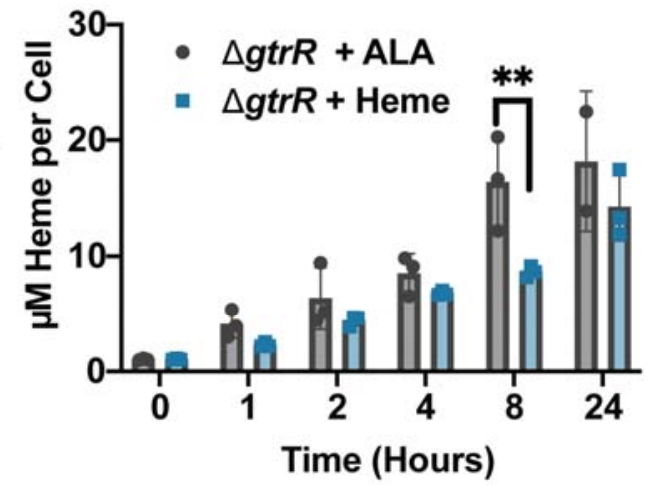

d.

KATG Activity
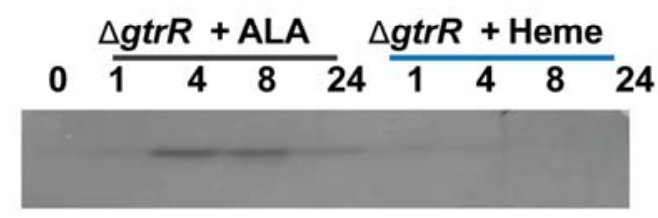

Time (Hours)

f.

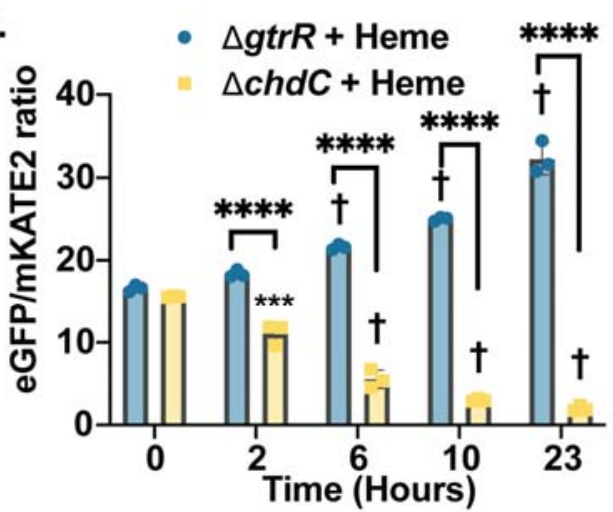

h.

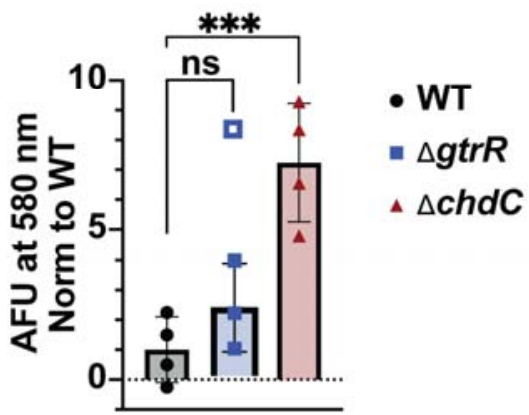

Fig. 1. Utilization of exogenous versus endogenous heme in Msm is regulated by ChdC. (a-d) Effects of ALA (5 $\mu \mathrm{g} / \mathrm{mL})$ or heme $(50 \mu \mathrm{M})$ supplementation on (a) growth 
rate, (b) total intracellular heme, (c) HS1-M7A detected labile heme, (d) and activity of the catalase-peroxidase KATG in $\Delta$ gtrR Msm cells. e-g Effects of heme $(50 \mu \mathrm{M})$ supplementation on (e) total intracellular heme, (f) HS1-M7A detected labile heme, (g) and activity of the catalase-peroxidase KatG in $\Delta g t r R$ vs $\Delta c h d C$ Msm cells. $\mathbf{h}$. Uptake of the heme analog zinc mesoporphyrin (ZnMP) in WT, $\Delta$ gtrR, and $\Delta c h d C \mathrm{Msm}$ cells cultured with $1 \mu \mathrm{M}$ ZnMP for 10 hours as quantified the ZnMP emission of ZnMP at 580 $\mathrm{nm}$. Duplicates from two independent trials are shown. In panel a, growth curves represent the average cell density of triplicate cultures. In panels $\mathbf{b}-\mathbf{c}$, data represent the mean \pm S.D. (error bars) of triplicate cultures. In panel $\mathbf{b}$, the statistical significance was assessed by two-way ANOVA with Bonferroni post hoc test: ${ }^{* *} p=0.0015$. In panel $\mathbf{c}$, the statistical significance was assessed by two-way ANOVA with Bonferroni post hoc test. Black asterisks denote statistically significant differences at each time point: ${ }^{* * * *} p$ $<0.0001$. Grey crosses denote statistically significant differences relative to "time 0 " for each set of treatments. $\dagger p<0.0001$. In all panels, differences that are not statistically significant are unlabeled. The zymogram depicted in panel $\mathbf{d}$ is representative of 2 independent trials. In panels e-f, data represent the mean \pm S.D. (error bars) of triplicate cultures. In panel e, the statistical significance was assessed by a two-way ANOVA with Bonferroni post hoc test: ${ }^{* * *} p<0.0001,{ }^{* *} p=0.0015$. In panel $\mathbf{f}$, the statistical significance was assessed by two-way ANOVA with Bonferroni post hoc test. Black asterisks denote statistically significant differences at each time point: ${ }^{* * * *} p<0.0001$. Grey crosses or asterisks denote statistically significant differences relative to "time 0" for each set of treatments: ${ }^{* * *} p=0.0001 ; \dagger p<0.0001$. In panel $\mathbf{h}$, the statistical significance was assessed by one-way ANOVA with Dunnett's post hoc test using WT Msm as control. For $\Delta g t r R$ the open square outlier was 1.4 standard deviations from the mean and was omitted from statistical analyses and ANOVA tests: ${ }^{* *} p=0.0009$; "ns" denotes non-significant differences and $p=0.2178$. In all panels, differences that are not statistically significant are unlabeled. The zymograms depicted in panels $\mathbf{d}$ and $\mathbf{g}$ are representative of 2 trials. 
a.

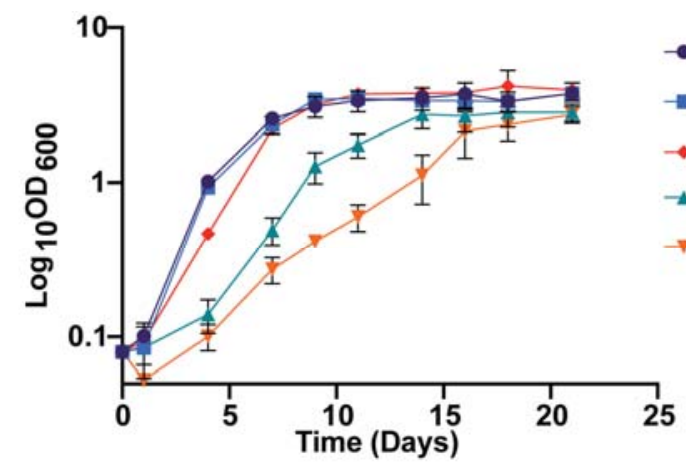

- WT

- WT + Heme

- $\triangle c h d C+$ Heme

- $\triangle g t r R+\mathrm{ALA}$

* $\Delta g$ trR + Heme b.

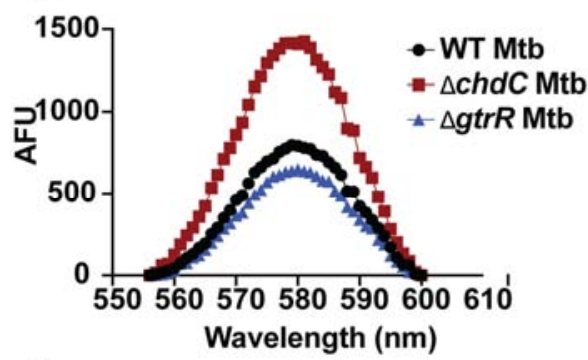

e.

WT Mtb $\frac{5 \mu \mathrm{M} \mathrm{Fe}}{1}{ }_{3} \frac{150 \mu \mathrm{M} \mathrm{Fe}}{12} 3$

g.

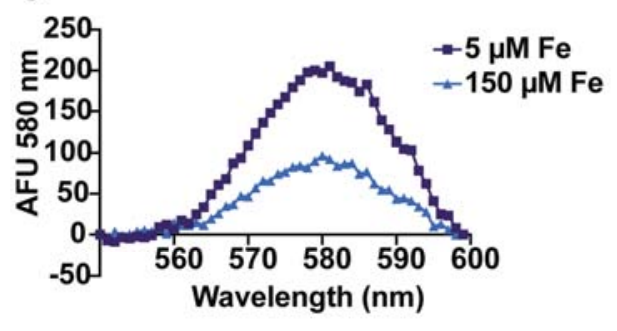

i.

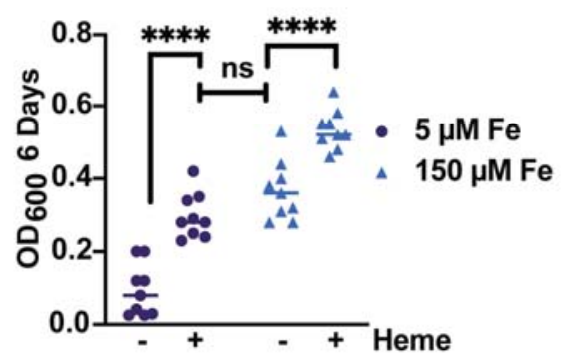

c.

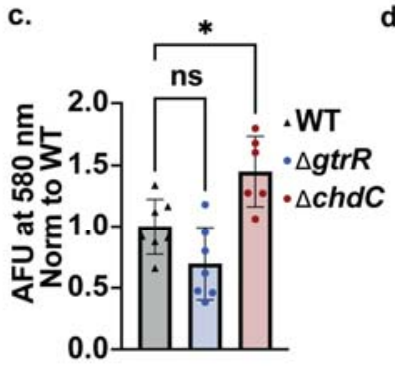

f.

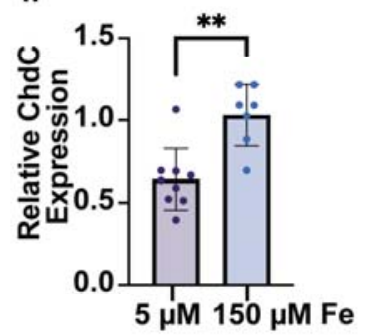

h.

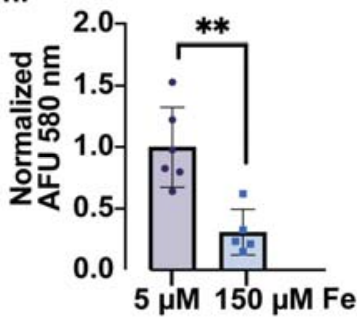

$\log _{2}$ Fold Change Relative to WT Mtb

d.

gtrR gsamuroD$c p f C-$ chd

mhuD

bvrR -

dppA-

$d p p D$

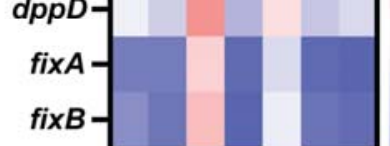

$-1$

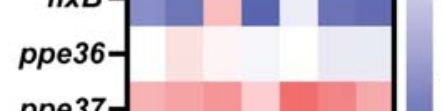

ppe37-

$m$

mmpl11-

ppe62-

bfrA-

bfr $B-$

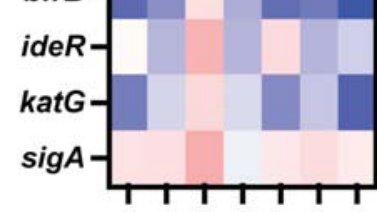

3

2

1

0

$-2$

$-3$ 
Fig. 2. ChdC is a negative regulator of exogenous heme utilization in Mtb. (a) Effects of ALA $(5 \mu \mathrm{g} / \mathrm{mL})$ or heme $(50 \mu \mathrm{M})$ supplementation on growth of WT, $\Delta$ gtrR, or $\triangle$ chdC Mtb strains. (b-c) Uptake of the heme analog zinc mesoporphyrin (ZnMP) in WT, $\Delta g t r R$, and $\Delta c h d C$ Mtb cells cultured with $1 \mu \mathrm{M}$ ZnMP for 48 hours as measured by fluorescence. (b) Representative average of 3 ZnMP fluorescence spectra from one experimental trial and (c) quantification of the ZnMP emission peak at $580 \mathrm{~nm}$ from replicates in three independent trials are shown. d. Heat map of $\log _{2}$ fold change of Mtb cultures compared to WT Mtb cultured in 7H9+ADS. Data shown is an average of two biological replicates. Xs represent $\log _{2}$ fold change of $<-6$ for genetic knockouts. (e) Representative western blot of ChdC expression in WT Mtb cultured in low (5 $\mu \mathrm{M} \mathrm{Fe}$ added) or high (150 $\mathrm{MM}$ Fe added) iron minimal media for 6 days. (f) Quantification of ChdC expression normalized to Ponceau $S$ total protein stain from replicates in 3 independent trials $n=9$ for $5 \mu \mathrm{M}$ and $\mathrm{n}=7$ for $150 \mu \mathrm{M}$. (g-h) Fluorescence detected uptake of the heme analog ZnMP in WT Mtb cells cultured in low $(5 \mu \mathrm{M})$ or high (150 $\mu \mathrm{M})$ iron minimal media for 6 days after exposure to $1 \mu \mathrm{M}$ ZnMP for 48 hours. (g) Representative ZnMP fluorescence spectra and (h) quantification of the ZnMP emission peak at $580 \mathrm{~nm}$ from replicates in two independent trials are shown, $\mathrm{n}=6$ for $5 \mu \mathrm{M}$ and $\mathrm{n}=5$ for $150 \mu \mathrm{M}$. i. Effects of heme $(12.5 \mu \mathrm{M})$ on growth of WT Mtb in minimal media (MM) supplemented with $5 \mu \mathrm{M}$ or $150 \mu \mathrm{M}$ Fe, $\mathrm{n}=9$. In panel a, growth curves represent the average cell density of triplicate cultures. In panel $\mathbf{c}$ the statistical significance was assessed by a one-way ANOVA with Dunnett's post hoc test using WT Mtb as control. In panel $\mathbf{c},{ }^{*} p=0.0152$ and "ns" denotes a non-significant $p=0.0911$. In panels $\mathbf{f}$ and $\mathbf{h}$, the statistical significance was assessed by a two-tailed unpaired t-test: (f) ${ }^{* *} p=0.0010$ and (h) $p=0.0023$. In panel $\mathbf{i}$, the statistical significance was assessed by using a twoway ANOVA with Bonferroni post hoc test: "ns" denotes a non-significant $p=0.2338$; ${ }^{* * *}$ $p<0.0001$; all unlabeled pairwise comparisons have $p<0.0001$ but are omitted for clarity. 


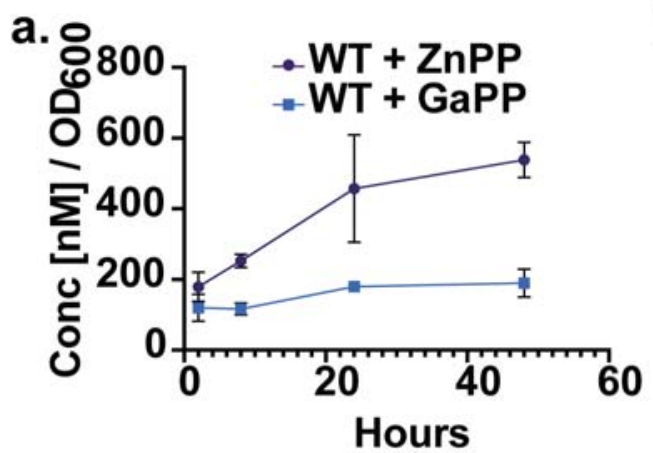

C.

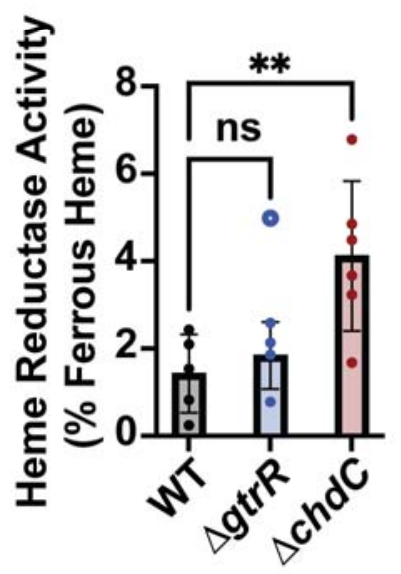

b.

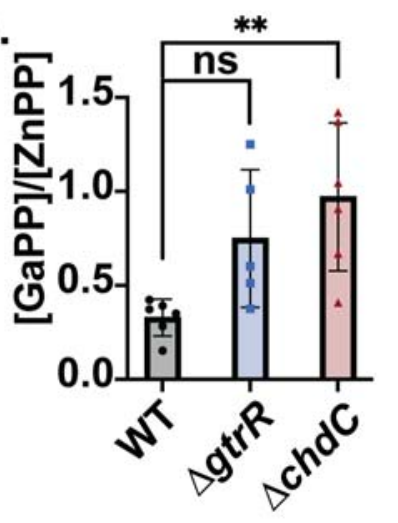

Figure 3. Mtb ChdC regulates reductive heme uptake. a. Binding and uptake of ZnPP and GaPP by WT Mtb measured over time by whole cell fluorescence. Concentrations were determined by standard curve. Data shown is from biological triplicates. $\mathbf{b}$. GaPP/ZnPP ratio of WT, $\Delta g t r R$ and $\Delta c h d C$ Mtb at 48 hours from two independent experiments. c. Percent ferrous heme at the cell surface of WT, $\Delta g t r R$ and $\Delta c h d C$ Mtb measured by pyridine hemochromogen. Data is shown as a ratio of ferrous to total heme and averaged from 2 independent trials. d. Effect of oxygen on exogenous heme bioavailability as measured by eGFP/mKATE2 ratio of HS1-M7A in WT Mtb in ambient air $\left(+\mathrm{O}_{2}\right)$ or an anerobic chamber $\left(-\mathrm{O}_{2}\right)$ for $\sim 18$ hours. Cells are in PBS $+/-5 \mu \mathrm{M}$ Heme at $30{ }^{\circ} \mathrm{C}$. For each independent experiment, ratio was normalized to the heme invariant sensor, HS1-M7A,H102A, to account for changes in fluorescence due to environment. To account for changes in fluorescence values between independent experiments, eGFP/mKATE2 ratios were normalized to Air $\left(+\mathrm{O}_{2}\right.$, - Heme). Data shown is from 3 independent experiments. For (b) a one-way ANOVA with Dunnett's post hoc comparison was used with WT as control, $p=0.0059$.For (c) a one-way ANOVA with Dunnett's post hoc comparison was used with WT as control. For $\Delta g t r R$ the open circle outlier was 1.6 standard deviations from the mean and was omitted from statistical analyses and ANOVA tests: ${ }^{* *} p=0.0092$. For $\left.(\mathbf{d})\right)$ a one-way ANOVA with Tukey's post hoc comparison was used, $p=0.0003$. In all panels, ns denotes not significant. 
a.

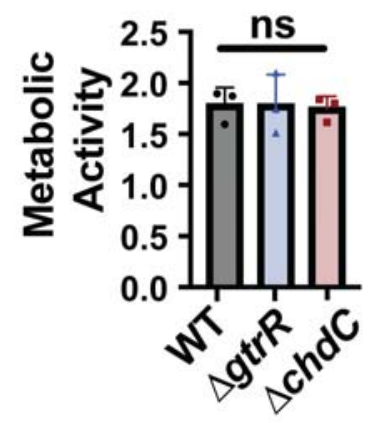

C.

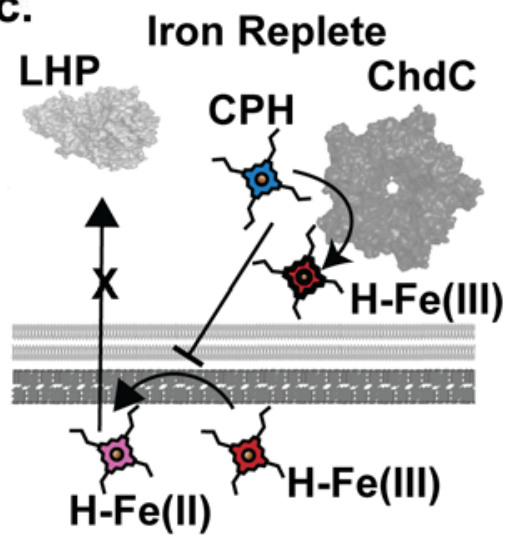

b.

\section{Post-Infection}

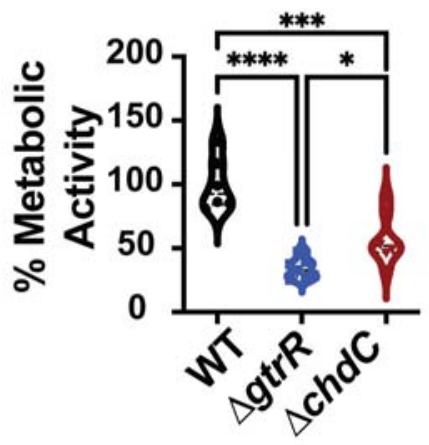

Iron Limited

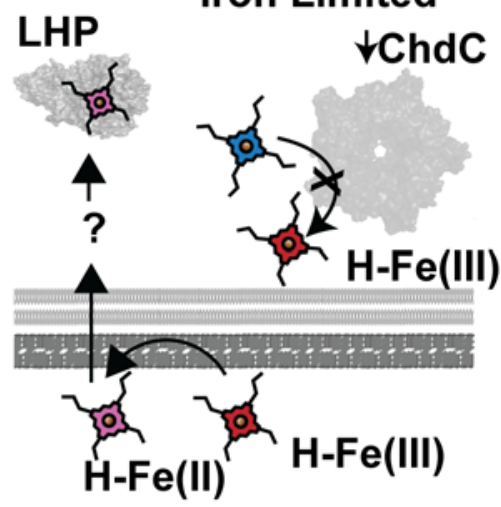

Fig. 4. Role of heme synthesis in a macrophage infection model. a. Formazan absorbance as turnover of MTT in WT Mtb, $\Delta$ gtrR Mtb and $\Delta$ chdC Mtb of $2 \times 10^{6}$ cells prior to macrophage infection. $2 \times 10^{6}$ cells are equivalent to $100 \%$ engulfment and survival in macrophage infection. b. MTT absorbance of WT Mtb, $\Delta$ gtrR Mtb and $\Delta$ chdC Mtb isolated from RAW 246.7 macrophages after 24 hours of infection from 2 independent trials. WT Mtb $\mathrm{n}=6, \Delta c h d C \mathrm{Mtb} \mathrm{n}=8$ and $\Delta g t r R \mathrm{Mtb} \mathrm{n}=7$. c. Proposed model for the roles of ChdC in coordinating heme synthesis, uptake, and utilization. LHP = labile heme pool, $\mathrm{CPH}=$ iron coproheme, $\mathrm{H}-\mathrm{Fe}(\mathrm{II})$ ferrous heme, $\mathrm{H}-\mathrm{Fe}(\mathrm{III})=$ ferric heme. In panel a, data represent the mean \pm S.D. (error bars) of triplicate cultures. The statistical significance was assessed by a one-way ANOVA with Dunnett's post hoc test using WT Mtb as a control: "ns" denotes a non-significant $p>0.9689$. In panel b, statistical significance was assessed by a one-way ANOVA with Tukey post hoc test: *** $p=0.0003$ (WT vs. ChdC $\Delta$ ); ${ }^{*} p=0.0423$ ( $\Delta$ chdC vs. $\left.\Delta g t r R\right) ;{ }^{* * *} p<0.0001$. 